\title{
Towards evaluation and prediction of building sustainability using life cycle behaviour simulation
}

\author{
Mohamed Marzouk ${ }^{1, *}$ and Mostafa El-hawary ${ }^{2}$ \\ ${ }^{1}$ Professor of Construction Engineering and Management, Structural Engineering Department, \\ Faculty of Engineering, Cairo University, Egypt. \\ ${ }^{2} \mathrm{PhD}$ Candidate, Structural Engineering Department, Faculty of Engineering, Cairo University, \\ Egypt.
}

\begin{abstract}
Nowadays researchers and practitioners are oriented towards questioning how effective are the different building life cycle activities contribution to preserving the environment and fulfilling the need for equilibrium. Terminologies such as Building sustainability and Green Buildings have long been adopted yet the evaluation of such has been driven through the use of rating systems. LEED of the United States, BREEAM of the United Kingdom, and Pearl of the United Arab Emirates are namely good examples of these rating systems. This paper introduces a new approach for evaluation of building life cycle sustainability through simulation of activities interaction and studying its behaviour. The effort focuses on comprehending impact and effect of suitability related activities over the whole building life cycle or period of time. The methodology includes gathering a pool of parameters through benchmarking of five selected rating systems, analytical factorization for the gathered parameters is used to elect the most influencing parameters. Followed by simulation modelling using System dynamics to capture the interaction of the considered parameters. The resulting behaviour obtained from simulation is studied and used in designing a tool for prediction of sustainability.
\end{abstract}

\section{Introduction}

The Buildings Sustainability has long been defined as the process of implementing an environmentally efficient resources management. Sometimes referred to as Green building with a structured approach where practice expands and complements the classical building design concerns of economy, utility, durability, and comfort [1]. Green building design involves finding the balance between homebuilding and the sustainable environment. This requires close cooperation of the design team, the architects, the engineers, and the client at all project stages [2]. Having a tool that can efficiently evaluate practicability and sustainability of buildings has been a vital concern by practitioners, and efforts for interventions resulted in using holistic concepts in introducing rating systems such as

\footnotetext{
* Correspoding author: mm_marzouk@yahoo.com
} 
Leadership in Energy and Environmental Design (LEED) of the United States, BREEAM of the United Kingdom, and Pearl of the United Arab Emirates.

Developed by the United States Green Building Council, LEED was one of the early attempts to tackle this evaluation issues, through setting rating systems for the design, construction, operation, and maintenance of green buildings. LEED, similar to most successive rating systems, has a main objective which is escalating reduction in the overall impact of the building processes on human health and the natural environment. However, beside this new technologies were constantly developed to enhance production of greener buildings. Rating systems nowadays forms a cornerstone in most industry projects; even owners are adopting its concepts as additional requirements. All rating systems efficiently evaluated environmental impact and resources use for an instance of the building in its life cycle as it is difficult to assess the relative improvements in sustainability of one decision versus another [3]. Studying the different activities interaction rather than individual influence could provide a different prospective fortunately systems thinking process facilitates such approach.

In concept, the goal of all rating systems is to produce sustainable buildings throughout the whole processes of construction, operation, and maintenance. From project idea generation and until destruction, while taking account for surrounding parameters like air, light, energy, water, construction materials and methods. While the applicability of each rating system differs, all rating systems draws upon occupants health, energy, and resources use, reducing waste, pollution and environmental degradation, and use of natural materials that are available locally. A wide range of mathematical techniques were developed to examine economic, environmental and ecological impacts of various pollution control actions, and thus aid decision makers in formulating effective sustainable development policies [4-10].

Systems thinking (System Dynamics) is defined as the process of understanding how things, regarded as systems, influence one another within a whole system, and fortunately has proved to be an efficient process for understanding the complex problems behaviour. The typical steps towards system dynamics involves primarily identification of the problem statement or question, followed by development of a dynamic hypothesis according to explanation of the problem causes. Then a system model is assembled with focus on reflecting behaviour in real world. With such model, insights are developed and conclusions are generated to enhance or propose solutions.

This paper investigates the use of system dynamics to evaluate building life cycle sustainability through behaviour of activities interaction. In other words, a way for comprehending impact and effect of sustainability related activates over whole building life cycle or period of time. In addition, the paper presents a structured approach towards interpreting building sustainability behaviour over its life cycle span, and paves the road to a proper debate over rating systems imposed activities.

\section{Proposed framework}

The proposed framework consists of four main steps as proposed by Forrester [11] with six supporting activities. The first step is "Problem Articulation (boundary selection)" which involves gathering information, on four worldwide rating systems. Within the same scope of problem articulation step, the parameters of the rating systems are then scaled in a table form through the first activity of "Parameters Selection". The resulting scale is then recorded as the base for benchmarking activity of the four rating systems to select a final list of potential parameters. The Following activity "Key Parameters Identification" involved parameters comparison with results from one of the most recent studies within the Middle East region that focused on selection of potential parameters for a new rating 
system introduced in the Kingdom of Saudi Arabia [12]. The output from this activity was based on identification of parameters with scores equal to 3 or 4.The second step was "Dynamic Hypothesis" through which correlation ideas for the key parameters were investigated. Using historical certification data reasonable correlation of the different parameters was derived. The following supporting activity involved mapping of the correlation ideas in a causal loop Diagram (CLD) form to properly grasp the understanding of the system. The Third step in the effort concerned "Formulation of Simulation Model" with all decision rules specification, and estimation of behavioural relationships, and consistency tests. The final step in this effort was interpreting the behaviour through "Policies Evaluation" which involved three activities "Behaviour Interpretation, Behaviour Analysis, and Adoption Rationale". A clear benefit for using system thinking approach was having feedback through closed loops as shown in Figure 1.

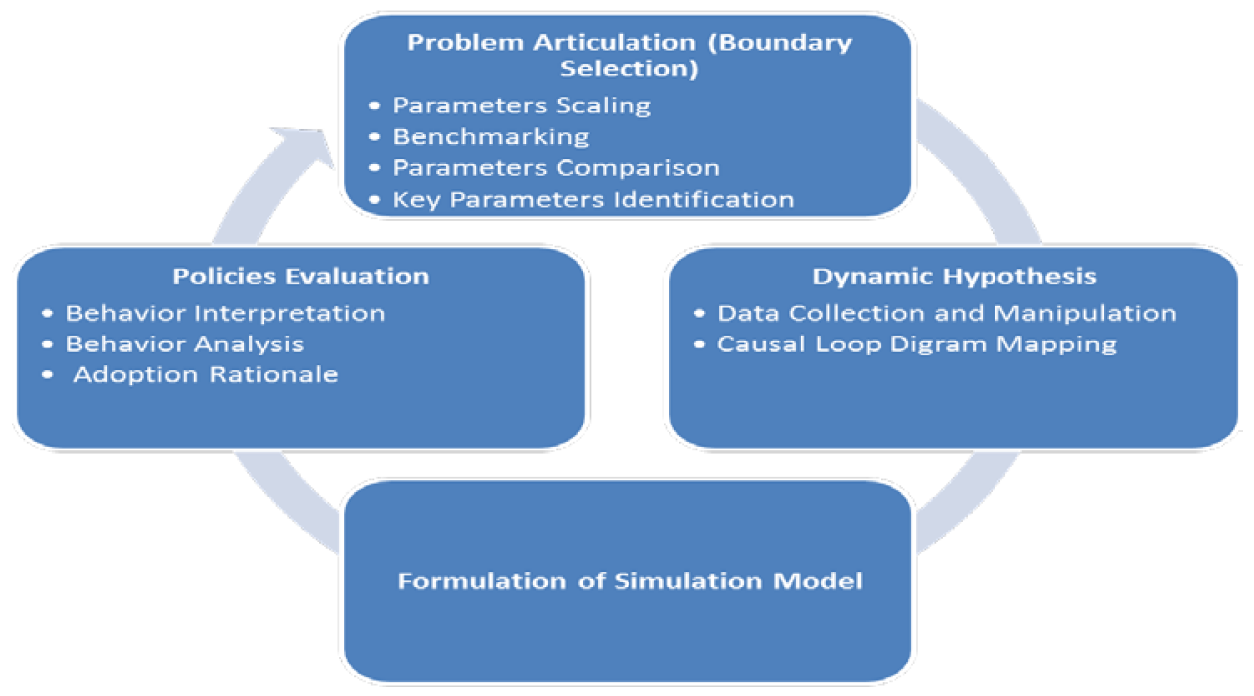

Fig. 1. Proposed building life cycle sustainability investigation framework.

\section{Problem articulation}

There are more than 600 rating system worldwide for evaluating the sustainability in construction industry (BRE 2008). The rating systems evaluate the sustainability of buildings through awarding points for satisfying green building criteria or parameters. The sustainability parameters and their importance weights usually differ from system to another according to differences in the environmental and regional conditions, and also due to cultural differences. Accordingly, each region needs to identify sustainability parameters that fit its conditions.

The sustainability rating systems that were considered in this study included are LEED, and BREEAM as an example from international rating systems. While for the regional examples GPRS and Pearl were selected. The aim of selecting such regional and international diverse rating systems helps in reflecting a wide range of environmental conditions.

Using simple approach for identification of parameters importance, each factor within context of named rating system is given a score only if exists in an alternative rating system. Accordingly the study boundaries were adopted from the selected rating systems factors available, and assumptions were made to consider the scaled parameters only as 
influencing factors. In order to equally evaluate parameters importance from each rating system, the sum of scores assigned in the previous sub-step was calculated. And a final table for parameters of interest was produced. Additionally, parameters were compared with results from one of the most recent studies within the Middle East region that focused on selection of potential parameters for a new rating system introduced in the Kingdom of Saudi Arabia [12].

\section{System dynamic modelling}

\subsection{Causal loop diagram}

One efficient method for interpretation of philosophies are the Causal loop Diagram (CLD) method, while most system thinking approaches deal with CLD as method of illustration it is also recorded as a mode of dilemma definition. The effort here involves mainly four loops as shown in Figure 2.

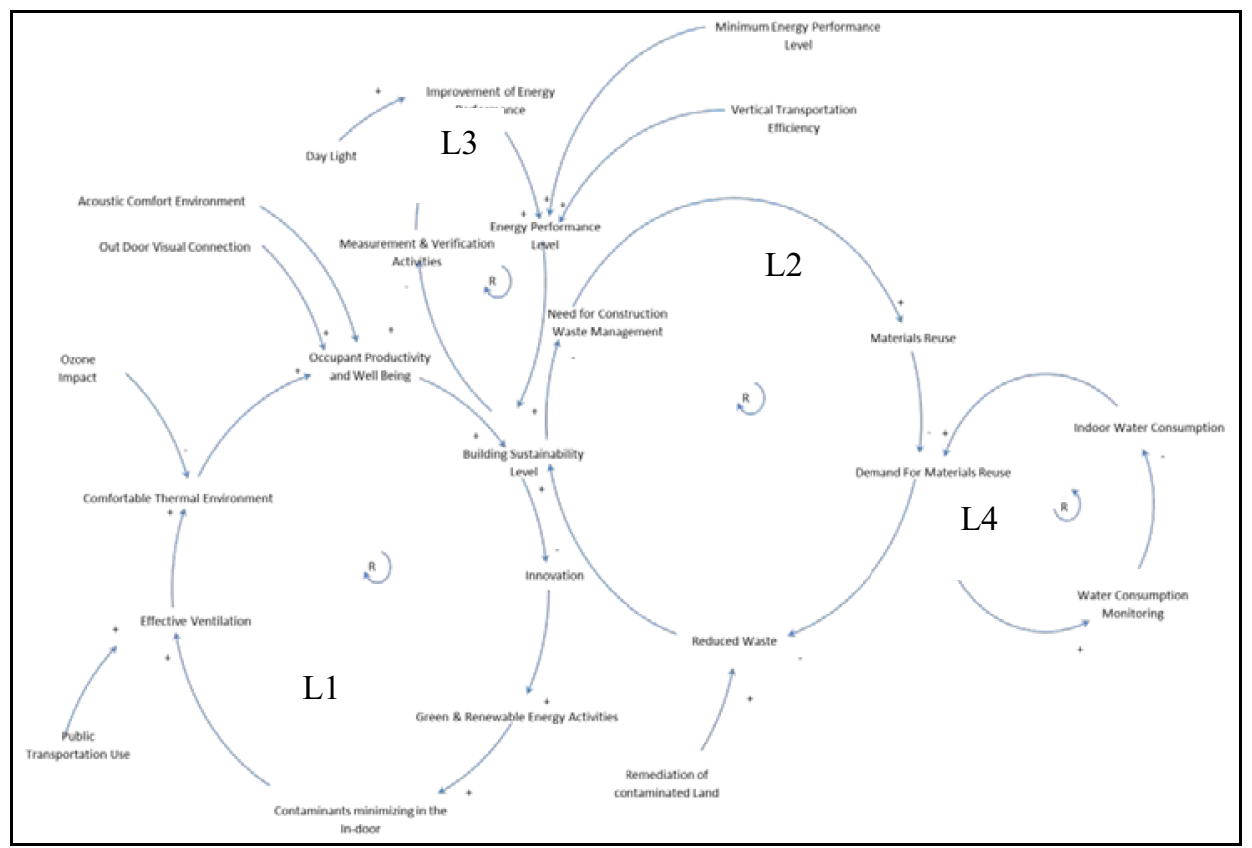

Fig. 2. Formulation of dynamic hypothesis using causal loop diagram.

\subsection{Formulation of simulation model}

Assumptions related to correlation ideas were made based on generated relations from the statistical analysis and assumed CLD. Due to complexity of studding all interaction between all the different key factors, the model has presented interactions assumed in the CLD. These interactions were: Correlation between occupants of the building and the different environmental impact factors, construction and maintenance activities dynamics, energy effect, and effect of water consumption.

Using VenSIM software the proposed simulation model was developed based on the resulting CLD and generated relationships from USGC data. Mainly the model was composed of five stocks with core stock of "Building sustainability level. Figure 3 
illustrates the model with all stocks in the model. The simulation runs were performed for 100 years as a proof of concept towards using the technique in addition to illustration of expected behaviour.

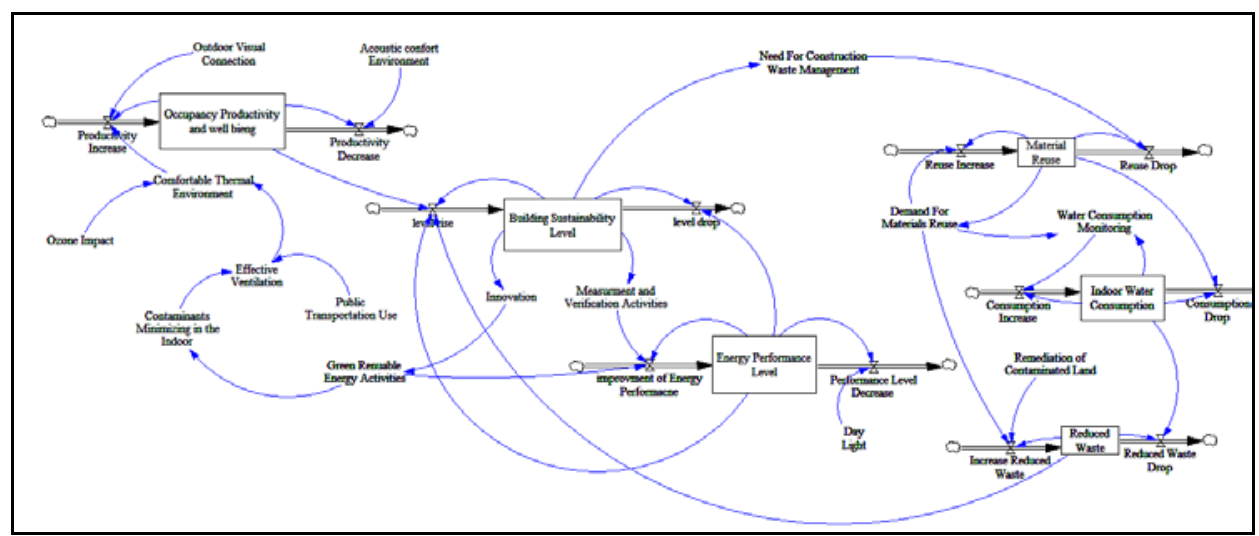

Fig. 3. Graphical representation of simulation model.

Conventionally through the construction period most sustainability activities and precautions are not yet implemented fully and accordingly behaviour hesitates, while through the early period of operation, sustainability plans will be well implemented resulting in a dramatic increase in behaviour, followed by less interest in enhancement of sustainability activities. The same conceptual understanding can be mapped over the rest of the illustrated behaviours. For instance, Figure 4-b illustrates the behaviour of Occupancy Productivity and Well Being (OPWB), from which the smooth increase can indicates how adoption of sustainability activities may results in better working environment. The behaviour also is recorded as a desired on for such factor. Additionally, Figure 4-c shows the behaviour of Indoor Water Consumption (IWC) which is, initially, has no water consumption record through construction stage, while a sudden rise of consumption occurs as soon as the building start its operation, followed by a continuous steady decrease in consumption as occupants continue applying sustainability techniques. On the other hand, Figure 4-d depicts Energy Performance Level (EPL) behaviour which starts with specific value and drops through period of construction due to sustainability measures and techniques implementation. As soon as the operation stage, starts a sudden increase in energy performance level occur. This may add to the increase in energy usage and accordingly this increase will drops gradually due to inadequate implementation of sustainability measures and techniques.

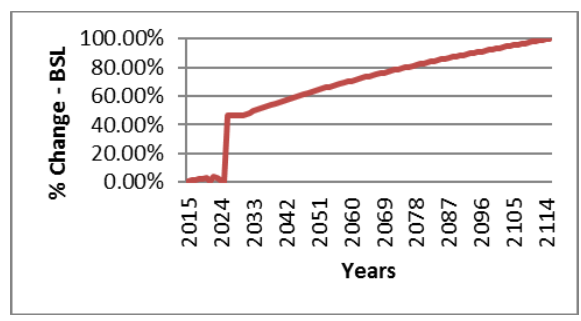

a. Building Sustainability Level Behaviour

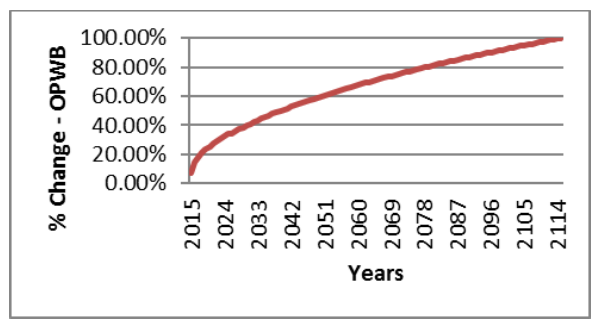

b. Occupancy Productivity and Well Being Behaviour 


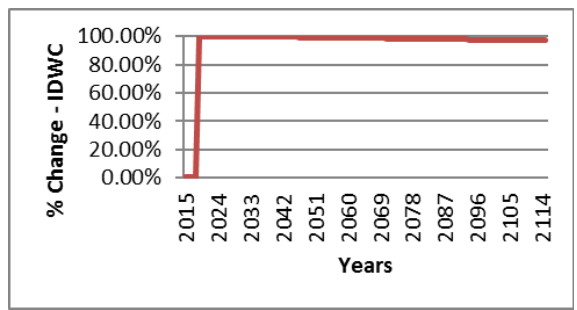

c. Indoor Water Consumption Behaviour

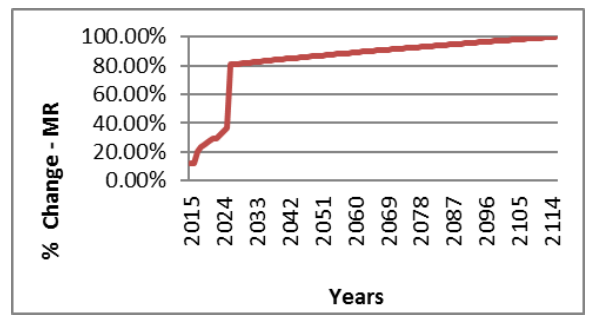

e. Materials Reuse

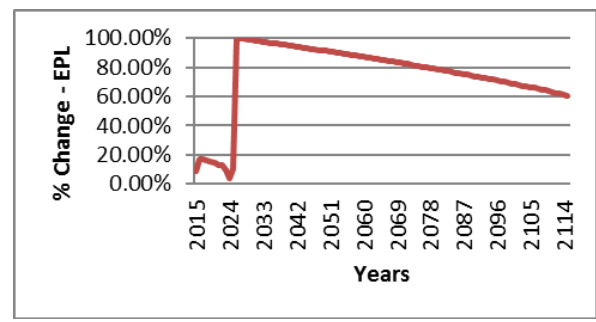

d. Energy Performance Level Behaviour

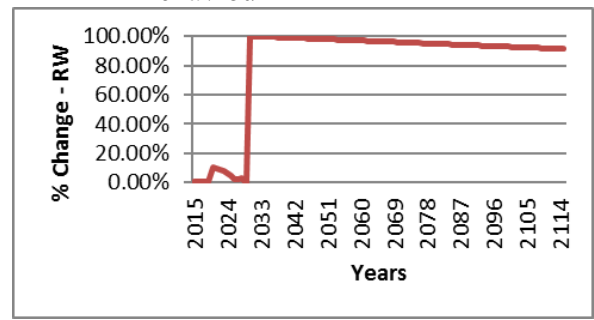

f. Reduced Waste

Fig 4. Building sustainability level behaviour.

\section{Summary and conclusion}

This research presented a generic framework towards implementing system thinking based approach for building life cycle sustainability enhancement. The effort methodology has included four main steps with six supporting activities, starting by problem articulation and moving to dynamic hypothesis, then formulation and modelling, and finally policies evaluation. One major contribution from implementing this effort over traditional holistic approaches was identifying the benefit of considering interaction between system factors. The benefit was clear in the spotted negative effect recorded by modelling building lifecycle sustainability behaviour. Moreover the use of system thinking in building life cycle comprehension added number of dimensions such as sustainability stretched analysis, factors interaction prospective, and possibility for performance prediction. Future enhancement for the framework shall involve lowering the threshold for parameters selection to score of 2 or less to increase the number of factors studied, also inclusion of additional causes correlations shall enhance the produced behaviour and will provide more robust model for policies recommendation. . An additional important fragment shall be the inclusion of "delay" for some factors that may cause burden shift after completion of the construction period.

\section{References}

1. EPA, Green Building Basic Information U.S. Environmental Protection Agency, (2009) available at $<$ http://www.epa.gov/greenbuilding/pubs/about.htm>.

2. Ji, Y., and Plainiotis, S. Design for Sustainability. China Architecture \& Building Press, Beijing, China (2006).

3. Anastas, P.T., and Zimmerman, J.B. Design Through the 12 Principles of Green Engineering. Environmental Science \& Technology, 37(5), 94A - 101A (2003). 
4. Zhang, N., Li, Y.P., Huang W.W., and Liu, J. An Inexact Two-Stage Water Quality Management Model for Supporting Sustainable Development in a Rural System. Journal of Environmental Informatics, 24(1) 52-64 (2014).

5. Chang, N.B., Chen, H.W., and Ning, S.K. Identification of river water quality using the fuzzy synthetic evaluation approach. Journal of Environmental Management, 63(3), 293-305 (2001).

6. Karmakar, S., and Mujumdar, P.P. A two-phase grey fuzzy optimization approach for water quality management of a river system. Advances in Water Resources, 30(5), 1218-1235 (2007).

7. Deviney Jr, F.A., Brown, D.E., and Rice, K.C. Evaluation of bayesian estimation of a hidden continuous-time Markov chain model with application to threshold violation in water-quality indicators. Journal of Environmental Informatics, 19(2), 70-78 (2012).

8. Graveline, N., Loubier, S., Gleyses, G., and Rinaudo J.D. Impact of farming on water resources: Assessing uncertainty with Monte Carlo simulations in a global change context. Agricultural Systems, 108, 29-41 (2012).

9. Kisi, O., Akbari, N., Sanatipour, M., Hashemi, A., Teimourzadeh, K., and Shiri, J. Modeling of dissolved oxygen in river water using artificial intelligence techniques. Journal of Environmental Informatics, 22(2), 92-101 (2013).

11. Xu, T.Y., and Qin, X.S. Solving water quality management problem through combined genetic algorithm and fuzzy simulation. Journal of Environmental Informatics, 22(1), 39-48 (2013).

12. Forrester, J. W. Industrial Dynamics, Wiley, New York. U.S (1961).

13. Al-Gahtani, K., AlSulahi, I., El-Hawary, M., and Marzouk, M. Investigating Sustainability Parameters of Administrative Buildings in Saudi Arabia. Technological Forecasting \& Social Change Journal, 105, pp. 41-48 (2016). 